\title{
Cost analysis of extended half-life recombinant factor IX products in the treatment of haemophilia B in Italy: an update
}

\author{
Andrea Aiello ${ }^{1}$, Maria E. Mancuso ${ }^{2}$, Serena Leone ${ }^{1}$, Letizia Rossi ${ }^{1}$, Lorenzo Cioni ${ }^{3}$, Cristina Teruzzi ${ }^{3}$ \\ ${ }^{1}$ Intexo Società Benefit S.r.l, Milano - Italy \\ ${ }^{2}$ Center for Thrombosis and Haemorrhagic Diseases, Humanitas Clinical and Research Center - IRCCS, Rozzano, Milan - Italy \\ ${ }^{3}$ Swedish Orphan Biovitrum, Milan - Italy
}

\begin{abstract}
Haemophilia B (HB) is a rare disease which may lead to chronic disabling arthropathy, resulting in a significant clinical, social and economic impact. In recent years, new extended half-life (EHL) factor IX concentrates produced by recombinant technology (rFIX) have been developed. They have shown significantly prolonged half-life as compared to other rFIX products and improved bleeding control when used as prophylaxis. To date, EHL $\mathrm{rFIX}$ products reimbursed in Italy are a recombinant coagulation factor IX produced with Fc technology (rFIXFc) and a recombinant fusion protein containing rFIX fused with recombinant albumin (rIX-FP). The results of extension studies with injection intervals with a median of almost every 14 days for the complete individualized interval prophylaxis (IP) group on rFIXFc and 21 days for a selected subgroup of patients on rIX-FP have recently been published.

The aim of this analysis was to estimate the cost of prophylactic treatment with rFIXFc and rIX-FP in adult patients, in the light of new clinical evidence and current average prices in Italy.

The cost of therapy was estimated on the basis of the results of extension studies, the average prices reported in regional drug tenders and assuming an average patient weight of $70 \mathrm{~kg}$.

The analysis estimated a cost per patient/year between $€ 224,407$ and $€ 230,355$ for $\mathrm{rFIXFc}$ and between $€ 242,259$ and $€ 368,587$ for rIX-FP. The sensitivity analysis confirmed the robustness of the results.

The use of rFIXFc over rIX-FP proves to be the least expensive choice for the treatment of HB in Italy.
\end{abstract}

Keywords: Cost analysis, Haemophilia B, Italy, Prophylaxis, rFIXFc

\section{Introduction}

Haemophilia $B(H B)$ is a rare disease characterized by a succession of haemorrhages mainly in joints and muscles, which may lead to chronic disabling arthropathy. In addition to presenting an important impact from a clinical point of view, the pathology affects the quality of life of patients, and the consumption of economic resources in terms of both direct and indirect healthcare costs $(1,2)$.

Received: August 9, 2021

Accepted: December 6, 2021

Published online: January 19, 2022

Corresponding author:

Andrea Aiello

Intexo Società Benefit S.r.l.

Via Fara 35

20124 Milano - Italy

andrea.aiello@intexo.it
HB is caused by a deficiency in coagulation factor IX (FIX); to date prophylactic administration of FIX concentrates is the gold standard of treatment. Recombinant FIX concentrates ( $r F I X)$ are the most widely used products $(3,4)$. In recent years, new extended half-life (EHL) FIX concentrates have been developed. EHL rFIX products have shown an improved pharmacokinetic profile with a significantly prolonged halflife compared with unmodified rFIX products and a better bleeding control when used according to prophylactic regimens (4).

To date, the EHL rFIX products reimbursed in Italy are: (a) rFIX covalently fused to the Fc domain of human immunoglobulin G1 (rFIXFc; eftrenonacog alfa [Alprolix ${ }^{\mathrm{TM}}$ ]); (b) a recombinant fusion protein containing rFIX fused with recombinant albumin (rIX-FP; albutrepenonacog alfa [Idelvion ${ }^{\mathrm{TM}}$ ]). These drugs have shown an increase in their use among patients with $\mathrm{HB}$ as a replacement therapy as compared with standard half-life (SHL) rFIX products in recent years (3).

In a recent publication, the impact on the healthcare budget in Italy as a result of an increased use of rFIXFc, as a 
prophylactic treatment, over rIX-FP and SHL rFIX concentrates (nonacog gamma and nonacog alfa) was assessed, estimating a saving of approximately $€ 2$ million over a 3-year period (1). This analysis estimated an average cost of $€ 225,185$ per adult patient per year for rFIXFc and $€ 312,327$ for rIX-FP, based on the dosages reported in the Summaries of Product Characteristics, literature data for bleeding frequency and management, and average prices from regional drug tenders. Sensitivity analysis confirmed the robustness of the results in favour of the treatment with rFIXFc (1).

Recently, extension clinical studies' results have been published $(5,6)$ confirming the positive benefit/risk profile of the two medicinal products.

The focus of the extension studies was to integrate the safety and efficacy profile (long-term) of the two EHL rFIX concetrates for routine prophylaxis and on-demand treatment of bleedings. Both studies $(5,6)$ enrolled previously treated adolescent and adult patients of different races from various geographic regions, although dose regimen and treatment intervals were different between the two trials $(5,6)$. Treatment intervals for rIX-FP prophylaxis dose were determined based on the parameters used in the pivotal study. Intervals of 7 days (35-50 IU/kg), 10 or 14 days $(50-75 \mathrm{lU} / \mathrm{kg}$ ) and 21 days $(100 \mathrm{lU} / \mathrm{kg}$ ) for patients $\geq 18$ years were used in Mancuso et al (5). For rFIXFc prophylaxis, as reported in Pasi et al, treatment intervals were: (a) weekly prophylaxis (20-100 IU/kg); (b) individualized interval prophylaxis (IP) (100 IU/kg every 8-16 days or twice monthly, with a median administration of 13.6 days); (c) modified prophylaxis (personalized on patient) (6).

\section{Objective}

The aim of this analysis was to estimate the cost of therapy with rFIXFc and rIX-FP in adult patients with $\mathrm{HB}$, given the updated clinical evidence and current average prices in Italy.

\section{Methods}

The methodology consists in a cost analysis of rIX-FP and rFIXFc treatments, based on results of the clinical extension trials $(5,6)$.

Average doses and frequencies of administration of EHL rFIX concentrates in the prophylactic regimen were derived from the clinical extension trials, as were the bleeding rates and the amount of product used to manage bleeding episodes $(5,6)$. The analysis considered the weekly administration regimens for both the products; the 21 days administration for rIX-FP and the IP regimen for rFIXFc. No indirect comparison or specific clinical analysis between trials was performed. Trial data were used only to estimate the EHL dosages according to different regimens $(5,6)$.

In the rFIXFc arm of the analysis, data associated with weekly prophylaxis and IP (median interval 13.6 days) were considered (6).

Indeed for rIX-FP, data associated with weekly dosing and with a maximum extension up to 21 days were used (5).

Drug costs were estimated on the basis of the average prices of $\mathrm{EHL}$ rFIX concentrates reported in drug tenders from 17 Italian regions (Abruzzo, Apulia, Basilicata, Calabria, Campania, Emilia Romagna, Friuli Venezia Giulia, Lazio, Liguria, Lombardy, Marche, Piedmont, Sardinia, Sicily, Tuscany, Umbria, Veneto; data on file).

To calculate the costs of prophylaxis and management of bleeding episodes, the following parameters, from clinical trials, were considered: bleeding rates, rFIX doses, number of administrations needed for prophylaxis and bleeding management, total dose in IU per $\mathrm{kg}$ per patient $(5,6)$. These unit quantities were multiplied by average patient weight of $70 \mathrm{~kg}$ (Tab. I). The total dosages per patient were then multiplied by the mean regional tender prices (unit prices), amounting to $1.21 € / I U$ for $r F I X F C$ and $1.98 € / I U$ for rIX-FP.

TABLE I - Mean annual consumption of rFIXFc and rIX-FP per patient in prophylaxis and management of bleeding episodes

\begin{tabular}{|c|c|c|c|c|c|c|}
\hline \multicolumn{2}{|l|}{ Variables } & \multicolumn{2}{|c|}{ rFIXFc } & \multicolumn{2}{|c|}{ rIX-FP } & \multirow[t]{2}{*}{ Reference } \\
\hline & & Weekly* & IP every 14 days*' & Weekly* & Every 21 days* & \\
\hline \multicolumn{7}{|c|}{ Unit Dose } \\
\hline Prophylaxis & Frequency adm per week & 1.00 & 0.50 & 1.00 & 0.33 & $(5,6)$ \\
\hline $\begin{array}{l}\text { Bleeding } \\
\text { management }\end{array}$ & IU/kg per adm & 51.80 & 36.60 & 56.36 & 56.36 & $(5,6)$ \\
\hline \multicolumn{2}{|c|}{ Patient weight $(\mathrm{kg})$} & 70 & 70 & 70 & 70 & Assumption \\
\hline \multicolumn{7}{|c|}{ Total Dose } \\
\hline Prophylaxis & IU per year & 176,540 & 184,912 & 180,908 & 121,091 & Estimate \\
\hline Bleeding & IU per year & 8,340 & 4,868 & 5,247 & 1,262 & Estimate \\
\hline
\end{tabular}

$\mathrm{ABR}=$ annualized bleeding rate; adm = administration; IP = individualized interval prophylaxis; IU = international units; $\mathrm{kg}=$ kilogram; $\mathrm{rFIXFC}=$ recombinant extended half-life factor IX produced with Fc technology; rIX-FP = recombinant fusion protein consisting of FIX with albumin moiety.

* Prophylactic administrations.

${ }^{\dagger}$ Individualized administration with a median interval of 13.6 days. Values (unit dose) are adjusted/calculated at 14 days. 
To test the robustness of the results, a univariate sensitivity analysis was developed in which a decrease in the price of rIX-FP of $-10 \%$ was considered. This theoretical discount was considered instead of standard deviation in regional prices, because there is no variability for rIX-FP and a very small one for rFIXFc ( $\pm 0.12 \%)$. In the sensitivity analysis, the costs of the two EHL rFIX concentrates were compared by assuming different treatment rates with rIX-FP, with administrations every 7 or 21 days, while only administration almost every 14 days, as in the IP regimen, was considered for rFIXFc (conservative assumption, because this is the most expensive regimen for rFIXFc) (6). Using a sample of 100 patients for each comparison arm, in what percentage of patients receiving rIX-FP every 21 days the two treatments would reach the same expenditure was calculated, considering a $10 \%$ discount for rIX-FP.

The cost analysis was carried out from the perspective of the National Healthcare Service (NHS).

\section{Results}

According to the clinical and consumption data shown in Table I, costs per patient in different regimens were estimated. Overall, as shown in Table II, the mean annual costs for treatment with rFIXFc were estimated between $€ 224,407$ (of which $€ 214,284$ was for prophylaxis) for weekly administration and $€ 230,355$ (€224,446) for administration almost every 14 days (median interval of 13.6 days). Mean annual treatment costs were higher for rIX-FP, ranging from $€ 242,259$ $(€ 239,760)$ for administration every 21 days to $€ 368,587$ $(€ 358,198)$ for weekly administration. Overall, it was estimated that potential savings from using rFIXFc instead of rIX-FP were $€ 11,904$, comparing rFIXFc almost every 14 days vs rIX-FP every 21 days, and $€ 144,180$, comparing weekly rFIXFc vs weekly rIX-FP.

With a $10 \%$ discount of the rIX-FP price, that is, $1.78 € / I U$, the annual cost for weekly treatment was still higher than that with rFIXFc, with an average cost of $€ 331,356$. Results show increased annual cost equivalent to $+€ 101,001$ vs rFIXFc almost every 14 days and $+€ 106,949$ vs rFIXFc for weekly administration. In contrast, a scenario with rIX-FP administered every 21 days was very similar to that with rFIXFc (both administration frequencies), with an average annual cost of $€ 217,789$. The results of the sensitivity analysis are described in Table II.

Taking the abovementioned scenario, which considers a price of $1.78 € / I U$ for rIX-FP, changes in expenditure in a hypothetical cohort of 200 adult patients of whom 100 were treated with rFIXFc almost every 14 days (conservative hypothesis) vs 100 treated with rIX-FP were estimated, with variable shares between weekly and 21-day therapy. The results are shown in Fig. 1. Overall, the analysis showed that with the increase in the proportion of patients treated with rIX-FP every 21 days, the total expenditure in that cohort would decrease as well as the differences vs the cohort treated with rFIXFc.

Figure 1 shows that in case $10 \%$ of 100 patients from the rIX-FP cohort receive administrations every 21 days the total cost will amount to $€ 32.0$ million vs $€ 23.0$ million in the rFIXFc cohort with a delta expenditure (rIX-FP - rFIXFc cohorts) of $+€ 8,964,460$ per 100 patients per year. Also considering that $50 \%$ of patients treated with rIX-FP had administrations

TABLE II - Mean treatment costs with rFIXFc e and rIX-FP per patient per year. Base case and sensitivity analysis

\begin{tabular}{|c|c|c|c|c|c|}
\hline \multirow[t]{2}{*}{ Variables } & \multicolumn{2}{|c|}{ rFIXFc } & \multicolumn{2}{|c|}{ rIX-FP } & \multirow[t]{2}{*}{ Reference } \\
\hline & Weekly* & IP every 14 days*' & Weekly* & Every 21 days* & \\
\hline \multicolumn{6}{|c|}{ Base Case Analysis } \\
\hline Base Case: Price per IU & $€ 1.21$ & $€ 1.21$ & $€ 1.98$ & $€ 1.98$ & Data on file \\
\hline \multicolumn{6}{|c|}{ Total Cost per Patient/Year } \\
\hline Prophylaxis & $€ 214,284$ & $€ 224,446$ & $€ 358,198$ & $€ 239,760$ & Estimate \\
\hline Bleedings & $€ 10,123$ & $€ 5,909$ & $€ 10,389$ & $€ 2,499$ & Estimate \\
\hline Total & $€ 224,407$ & $€ 230,355$ & $€ 368,587$ & $€ 242,259$ & Estimate \\
\hline \multicolumn{6}{|c|}{ Sensitivity Analysis } \\
\hline Sensitivity Analysis: Price per IU & $€ 1.21$ & $€ 1.21$ & $€ 1.78$ & $€ 1.78$ & Estimate \\
\hline \multicolumn{6}{|c|}{ Total Cost per Patient/Year } \\
\hline Prophylaxis & $€ 214,284$ & $€ 224,446$ & $€ 322,016$ & $€ 215,541$ & Estimate \\
\hline Bleedings & $€ 10,123$ & $€ 5,909$ & $€ 9,340$ & $€ 2,248$ & Estimate \\
\hline Total & $€ 224,407$ & $€ 230,355$ & $€ 331,356$ & $€ 217,789$ & Estimate \\
\hline
\end{tabular}

$I P$ = individualized interval prophylaxis; IU = international units; rFIXFc = recombinant coagulation factor IX Fc fusion protein; rIX-FP = recombinant fusion protein consisting of FIX with albumin moiety.

*Prophylactic administrations.

${ }^{\dagger}$ Individualized administration with a median interval of 13.6 days. Values (unit dose) are adjusted/calculated at 14 days. 


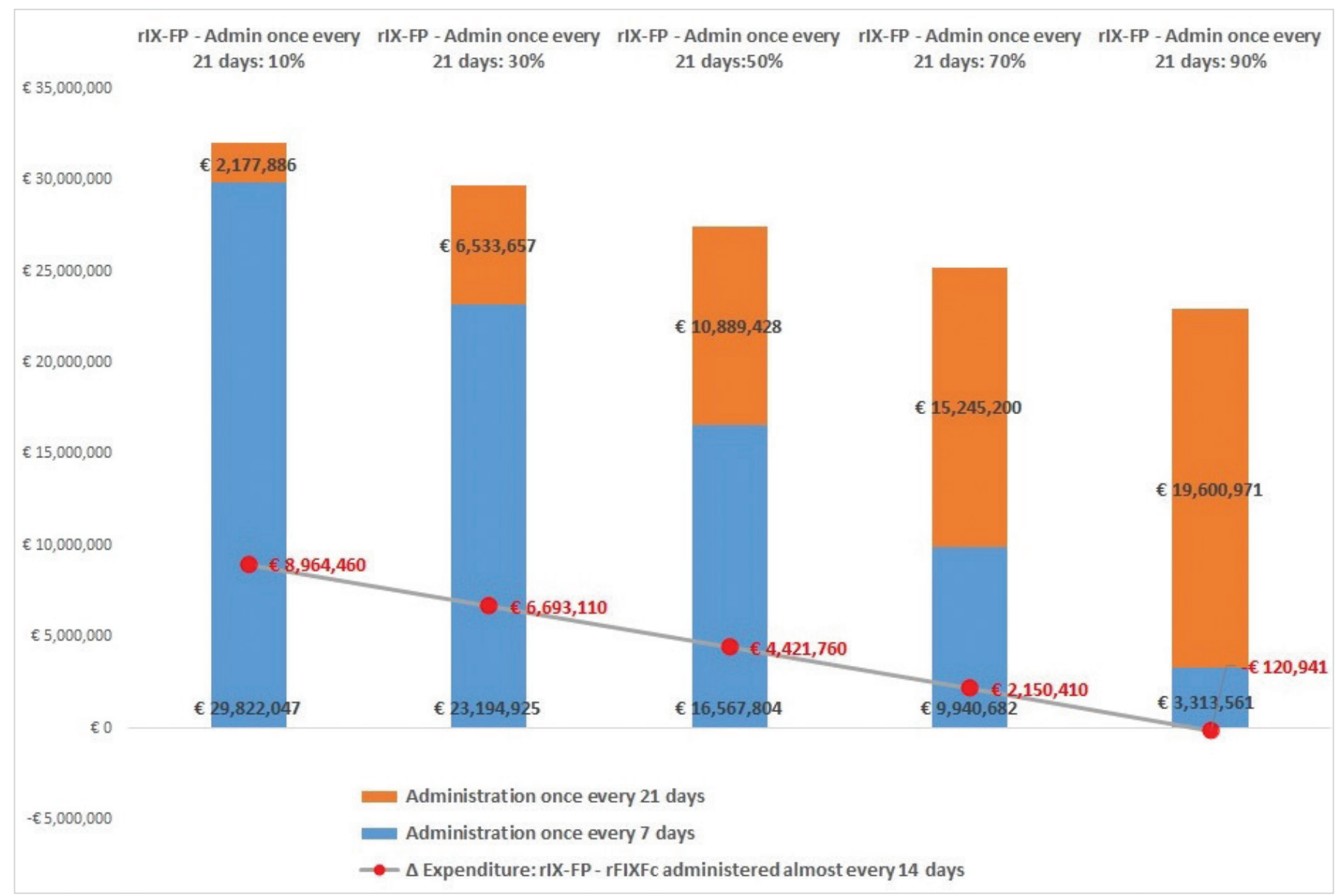

Fig. 1 - Sensitivity analysis: change in spending and savings considering a 10\% discount on the rIX-FP price and percentage of patients treated with rIX-FP every 7 or 21 days.

rFIXFc = recombinant coagulation factor IX Fc fusion protein; rIX-FP = recombinant fusion protein consisting of FIX with albumin moiety.

every 21 days, this cohort shows additional expenditure of $+€ 4,421,760$ vs rFIXFc administered almost every 14 days.

Results show that rIX-FP would achieve parity cost vs rFIXFc (with administration almost every 14 days) only when at least $89 \%$ of patients were treated with administration every 21 days. It should be noted that if a comparison with rFIXFc on a weekly basis was considered, the percentage of patients treated with rIX-FP every 21 days would amount to $94 \%$.

\section{Discussion}

The previous article mainly considered dosages in prophylaxis from the Summary of Product Characteristics (1), whereas the present analysis considered dosages and bleeding rates from the extension clinical trials $(5,6)$. Despite the clinical studies details, the results are very similar, highlighting a significant burden of prophylaxis on the overall cost of treatment, ranging from $95 \%$ to $99 \%$ of the total cost.

The results also confirm that rFIXFc (given current prices) is the least expensive drug for the NHS, while ensuring effective control of bleeding, with an annualized bleeding rate $(A B R)$ of $1.90 / 2.30$ which is lower or in line with previous published data (for both EHL rFIXs), but slightly higher than rIX-FP in the current analysis $(1,5,6)$.

It is important to highlight that our estimation was conservative due to significant differences in study designs, which resulted in a stepwise selection of suitable patients for longer administration intervals with rIX-FP and rFIXFc $(5,6)$. Anyhow, the ABR differences and bleeding management, from an economic point of view, have a minimum impact on total costs from $4.5 \%$ for rFIXFc weekly to $1.0 \%$ for rIX-FP (Tab. II).

Given the current prices, even if $100 \%$ of patients were treated with rIX-FP every 21 days during the year, this cohort would not reach neutral cost with rFIXFc, which remains the least expensive treatment and most economically advantageous choice for the Italian NHS. The analysis also shows that rIX-FP will reach parity cost with rFIXFc (administered almost every 14 days) only if it has a $10 \%$ discount and at least $89 \%$ of patients are treated every 21 days.

It is also very relevant that in the extension study of rIX-FP only 9 out of 59 patients (i.e. about 15\%) were successfully treated with the 21 days prophylactic regimen (5), which is distant from the above-mentioned $89 \%$ estimated in our study. Differently, it must be highlighted that the 
annual prophylactic mean costs of weekly administration and IP regimen with rFIXFc are very similar (6). Additional realworld data are needed to evaluate the percentage of patients treated with rIX-FP every 21 days, which, in any case, has shown a higher mean annual cost than rFIXFc, considering the actual prices of rFIX products.

Finally, it is relevant to mention that in a previous post hoc analysis, Shapiro et al studied long-term experience with extended interval dosing of rFIXFc using data up to the second data cut of B-YOND study and reported a subgroup of patients (6 out of 22), who had dosing intervals of 15-21 days (7). Nevertheless, if these rFIXFc dosing intervals were considered in our short communication, the results would still be in favour of rFIXFc.

\section{Conclusions}

Even in the light of new clinical evidence, which considers extended dosing intervals for both drugs, the use of rFIXFC over rIX-FP in the treatment of $\mathrm{HB}$ is still an economically advantageous choice for the Italian NHS, with overall savings, per patient per year, of about $€ 12,000$, comparing the extended administration of rFIXFc vs rIX-FP and $€ 144,000$ comparing the weekly ones. These results are confirmed by the sensitivity analyses.

\section{Disclosures}

Conflict of interest: a) AA, SL and LR are employees of Intexo Società Benefit S.r.l.; b) MEM reports personal fees from Swedish Orphan Biovitrum (Sobi), Bayer Healthcare, Biomarin, Catalyst Biosciences, CSL Behring, Grifols, Kedrion, LFB, Novo Nordisk, Octapharma, Pfizer,
Roche, Sanofi, Spark Therapeutics, UniQure, Takeda; c) LC and CT are employees of Sobi S.r.l., Milan, Italy.

Financial support: Sobi S.r.l., Milan, Italy, has provided financial support to cover the cost of this project and the editorial assistance, but has not influenced the manuscript content.

\section{References}

1. Aiello A, Mancuso ME, Colombo A, Teruzzi C, Berto P. Budget impact analysis of extended half-life recombinant factor IX (rFIXFc) in the treatment of haemophilia B. Glob Reg Health Technol Assess 2020;7(1):40-49. CrossRef

2. Kodra Y, Cavazza M, Schieppati A, et al. The social burden and quality of life of patients with haemophilia in Italy. Blood Transfus. 2014;12 Suppl 3:s567-s575. CrossRef

3. Abbonizio F, Arcieri R, Associazione Italiana Centri Emofilia (AICE) e Giampaolo A. Registro Nazionale Coagulopatie Congenite. Rapporto 2018. Roma: Istituto Superiore di Sanità; 2020. (Rapporti ISTISAN 20/14). Online

4. Srivastava A, Santagostino E, Dougall A, et al; WFH Guidelines for the Management of Hemophilia panelists and co-authors. WFH guidelines for the management of hemophilia. 3rd edition. Haemophilia. 2020;26(S6)(suppl 6):1-158. CrossRef PubMed

5. Mancuso ME, Lubetsky A, Pan-Petesch B, et al. Long-term safety and efficacy of rIX-FP prophylaxis with extended dosing intervals up to 21 days in adults/adolescents with hemophilia B. J Thromb Haemost. 2020;18(5):1065-1074. CrossRef PubMed

6. Pasi KJ, Fischer K, Ragni M, et al. Long-term safety and sustained efficacy for up to 5 years of treatment with recombinant factor IX Fc fusion protein in subjects with haemophilia B: results from the B-YOND extension study. Haemophilia. 2020;26(6):e262e271. CrossRef PubMed

7. Shapiro AD, Pasi KJ, Ozelo MC, et al. Extending recombinant factor IX Fc fusion protein dosing interval to 14 or more days in patients with hemophilia B. Res Pract Thromb Haemost. 2018; 3(1):109-113. CrossRef PubMed 